\title{
Carbon stars in the outer spheroid of NGC $6822^{\star, \star \star}$
}

\author{
S. Demers ${ }^{1}$, P. Battinelli ${ }^{2}$, and E. Artigau ${ }^{1}$ \\ 1 Département de Physique, Université de Montréal, CP 6128, Succursale Centre-Ville, Montréal, Qc, H3C 3J7, Canada \\ e-mail: [demers; artigau] aastro.umontreal.ca \\ 2 INAF, Osservatorio Astronomico di Roma Viale del Parco Mellini 84, 00136 Roma, Italy \\ e-mail: battinel@oarhp1.rm.astro.it
}

Received 26 April 2006 / Accepted 12 June 2006

\section{ABSTRACT}

\begin{abstract}
Context. From a $2^{\circ} \times 2^{\circ}$ of NGC 6822 survey we have previously established that this Local Group dwarf irregular galaxy possesses a huge spheroid having more than one degree in length. This spheroid is in rotation but its rotation curve is known only within $\sim 15^{\prime}$ from the center. It is therefore critical to identify bright stars belonging to the spheroid to characterize, as far as possible, its outer kinematics.

Aims. We use the new wide field near infrared imager CPAPIR, operated by the SMARTS consortium, to acquire $J, K_{\mathrm{s}}$ images of two $34.8^{\prime} \times 34.8^{\prime}$ areas in the outer spheroid to search for C stars.

Methods. The colour diagram of the fields allows the identification of $192 \mathrm{C}$ stars candidates but a study of the FWHM of the images permits the rejection of numerous non-stellar objects with colours similar to $\mathrm{C}$ stars.

Results. We are left with 75 new $\mathrm{C}$ stars, their mean $K_{\mathrm{s}}$ magnitude and mean colour are similar to the bulk of known NGC $6822 \mathrm{C}$ stars.

Conclusions. This outer spheroid survey confirms that the intermediate-age AGB stars are a major contributor to the stellar populations of the spheroid. The discovery of some $50 \mathrm{C}$ stars well beyond the limit of the previously known rotation curve calls for a promising spectroscopic follow-up to a major axis distance of $40^{\prime}$.
\end{abstract}

Key words. galaxies: individual: NGC 6822 - galaxies: structure - stars: carbon - galaxies: dwarf

\section{Introduction}

NGC 6822, a Local Group dwarf irregular galaxy, has been the first extragalactic object identified by Hubble (1925) who determined its distance to be $214000 \mathrm{pc}$ or $(m-M)=21.65$. NGC 6822 is located in the constellation Sagittarius at a low Galactic latitude $\left(\ell=25^{\circ}, b=-18^{\circ}\right)$. It is thus seen behind a relatively heavy stellar foreground and its reddening is far from negligible. Today, we adopt for NGC 6822 a distance of $(m-M)_{0}=23.35$, based on the RR Lyrae observations of Clementini et al. (2003), the Cepheids observations of Pietrzyński et al. (2004) and the near infrared observation of the tip of the red giant branch (TRGB) by Cioni \& Habing (2005). At $470 \mathrm{kpc}$, it is the nearest Magellanic-type galaxy, after the Magellanic Clouds.

Contrasting strongly with its discovery description by Barnard (1885) who depicted it as: “a 2 ' nebula, diffuse and difficult to see with his 5-inch instrument", NGC 6822 has been found to be much more extended. Its global structure was first studied by Hodge (1977) who described the bar as a $8^{\prime}$ long structure with a position angle of $10^{\circ}$. In a more detailed

* This publication makes use of data products from the Two Micron All Sky Survey, which is a joint project of the University of Massachusetts and the Infrared Processing and Analysis Center/California Institute of Technology, funded by the National Aeronautics and Space Administration and the National Science Foundation.

$\star \star$ Full Tables 2 and 3 are only available in electronic form at the CDS via anonymous ftp to cdsarc.u-strasbg. fr (130.79.128.5) or via http://cdsweb.u-strasbg.fr/cgi-bin/qcat? J/A+A/456/905 investigation, by Hodge et al. (1991), the galaxy could be traced to $10^{\prime}$. They adopt the point of view that the galaxy is circular rather than following the shape of the bar. We realize today that the low-density of the periphery of the galaxy can easily be masked by the substantial foreground density seen along the line of sight.

The first use of a wide-field imager, to search for C stars, by Letarte et al. (2002) has revealed that NGC 6822 is surrounded by a huge stellar structure extending to at least $20^{\prime}$ from its center and containing a substantial intermediate-age population. This huge spheroid was also seen by Lee \& Hwang (2005). Young stars were identified far from the center, along the HI disk by Komiyama et al. (2003). Recently, Battinelli et al. (2006) have mapped the elliptical spheroid of NGC 6822 to a semi-major axis distance of $36^{\prime}$. From radial velocities of numerous $C$ stars, Demers et al. (2005a) have shown that the spheroid is dynamically decoupled from the HI disk and rotates at right angles to it. Since C stars used as kinematical probes were then known only to a distance of $\sim 15^{\prime}$, it is of great interest to identify other $\mathrm{C}$ stars further out in the spheroid to extend its rotation curve and compare it to the HI rotation curve, observed to $\sim 30^{\prime}$ (Weldrake et al. 2003).

Few near-infrared investigators have targeted NGC 6822. Elias \& Frogel (1985) have obtained $J, K$ magnitudes of 18 mostly spectroscopically confirmed red supergiants, with a $\langle K\rangle=13$. These stars are much brighter than $\mathrm{C}$ stars. Davidge (2003) has observed three tiny areas near the NGC 6822 bar to determine the metallicity from the slope of the RGB. He concluded that the bulk of the NGC 6822 red giants are of intermediate-age rather than $\sim 12$ Gyr. The wide-field survey of 
Table 1. NGC 6822 observations with exposure times.

\begin{tabular}{lccc}
\hline \hline Field & RA J2000 Dec & $J$ & $K_{\mathrm{s}}$ \\
\hline NE & $19: 46: 34.1-14: 38: 21$ & $6846 \mathrm{~s}$ & $10017 \mathrm{~s}$ \\
SW & $19: 43: 25.8-14: 58: 38$ & $6092 \mathrm{~s}$ & $7304 \mathrm{~s}$ \\
\hline
\end{tabular}

a $20^{\prime} \times 20^{\prime}$ central region of NGC 6822 by Cioni \& Habing (2005) is a first attempt to present a global view of the AGB of NGC 6822 in the near infrared. More recently, Kang et al. (2006) have obtained $J, H, K$ photometry of small areas near the center, where the space density of $\mathrm{C}$ stars is quite high.

We then present results of a near-infrared survey, which targets the outer spheroid and takes advantage of the new widefield imager CPAPIR. It is essentially complementary to Cioni \& Habing (2005) observations. We survey two regions in the outer spheroid of NGC 6822 with a small overlap with their central field.

\section{Observations}

The $J, K_{\mathrm{s}}$ observations described here were obtained in service mode (from April to September 2005) with the CPAPIR (Caméra Panoramique Proche InfraRouge, see Artigau et al. 2004) imager attached to the CTIO $1.5 \mathrm{~m}$ telescope and operated by the SMARTS consortium. CPAPIR is based on a $2048 \times 2048$ Hawaii-2 infrared array detector. With a pixel size of $1.02^{\prime \prime}$, at the $1.5 \mathrm{~m}$ telescope it has a field of view of $34.2^{\prime} \times 34.2^{\prime}$. One frame consists of five co-added exposures of $5.4 \mathrm{~s}$ for $K_{\mathrm{s}}$ and a single $24.3 \mathrm{~s}$ for $J$. The script, for each observation, acquires 100 frames while introducing a $5^{\prime \prime}$ dither after each frame. The sky image is built up by running a median of nine frames, centered on the frame of interest. Stars are masked during the sky construction. These 100 frames are registered and a median image is built.

The combined median images are then analysed with DAOPHOT-II (Stetson 1994). The instrumental magnitudes and colours are transformed into the $J, K_{\mathrm{s}}$ system by crossidentifying our program stars with the 2MASS stars in the fields. Nearly one thousand 2MASS stars are seen in each field. Least square solutions, omitting the saturated bright stars and the faint 2MASS stars with poorer photometry, yield:

$$
\begin{aligned}
\mathrm{NE} J & =-3.686( \pm 0.046)+1.005( \pm 0.003) j_{\text {inst }} \\
\mathrm{NE} K_{\mathrm{s}} & =-3.972( \pm 0.069)+0.993( \pm 0.004) k_{\text {inst }} \\
\mathrm{SW} J & =-3.703( \pm 0.041)+1.0064( \pm 0.002) j_{\text {inst }} \\
\mathrm{SW} K_{\mathrm{s}} & =-4.262( \pm 0.070)+1.002( \pm 0.004) k_{\text {inst. }}
\end{aligned}
$$

These equations show that, as expected, the slopes are 1.00, we need only to apply the magnitude zero point shifts.

Figure 1 shows a SDSS image of NGC 6822 along with the outer ellipse (semi-major axis of $36^{\prime}$ ), defining the spheroid, identified by Battinelli et al. (2006). The two observed CPAPIR fields, we called NE and SW, are outlined. Their coordinates are given in Table 1 along with the exposure times, in seconds, for each filter.

\section{Results}

The colour-magnitude diagram of the combined two fields is presented in Fig. 2. Some 18000 stars with colour errors $\sigma_{j k}<$ 0.20 are plotted. A global reddening of $E(B-V)=0.25$ is

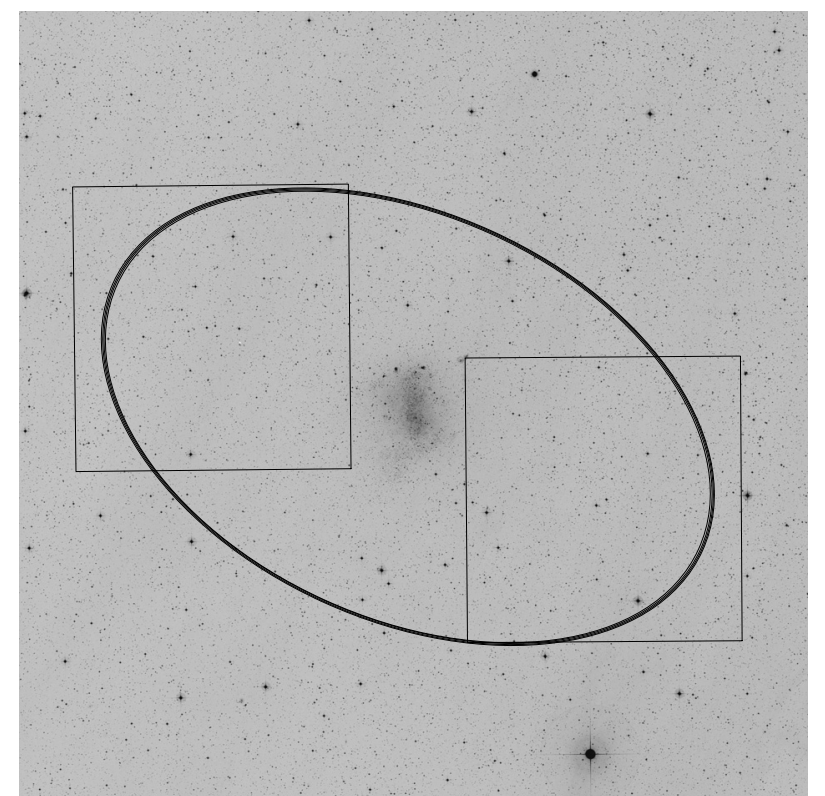

Fig. 1. NGC 6822, the outer $36^{\prime}$ ellipse is sketched along with the two CPAPIR fields, NE at the upper left and SW at the lower right, discussed in this paper.

assumed (van den Bergh 2000; Battinelli et al. 2006). Using the Schlegel et al. (1998) relations, this reddening translates into $E(J-K)=0.13$ and $A_{K}=0.09$, small but nevertheless not negligible values. The vertical line, drawn on the CMD at $\left(J-K_{\mathrm{s}}\right)=1.49$ corresponds to the adopted separation of O-rich and C-rich AGB stars, $\left(J-K_{\mathrm{s}}\right)_{0}=1.36$ by Cioni \& Habing (2005). The horizontal dashed line is the level of the TRGB, at $K_{\mathrm{s}}=17.1$ (Cioni \& Habing 2005). Our 360 stars in common with their photometry, (for which their $\sigma_{k}<0.20$ ) yield: $\Delta_{K_{\mathrm{s}}}=-0.06 \pm 0.32$, while our 185 stars (for which their $\left.\sigma_{j k}<0.30\right)$ yields: $\Delta_{J-K}=-0.05 \pm 0.24$. Our observed regions do not overlap with the near infrared photometry recently published by Kang et al. (2006).

Our CMD should be compared to Cioni \& Habing (2005) Figs. 7 and 10. However, our CMD contains a much larger contribution from the foreground/background because the areas observed are more than twice as large as their region and, most importantly because we target the outer parts of the galaxy where the proportion of NGC 6822 stars is low. The two major vertical features seen on the CMD are Galactic G dwarfs, on the left and the Galactic M dwarfs at $\left(J-K_{\mathrm{s}}\right) \approx 1.0$. Actually, since our photometry reaches barely magnitudes fainter than the tip of the red giant branch, the minority of the stars seen here belongs to NGC 6822.

\subsection{Distinction between stellar and non-stellar objects}

We see, in Fig. 2, 192 objects with magnitudes and colours corresponding to AGB C stars. They have $\left(J-K_{\mathrm{s}}\right)>1.49$ and are well above the TRGB. This represents an unexpectedly large number, when compared to the spheroid density profile, as determined by Battinelli et al. (2006). Pollution by background galaxies has been noticed by Battinelli et al. (2006) in the $\left(r^{\prime}-i^{\prime}\right)$ vs. $\left(g^{\prime}-r^{\prime}\right)$ colour-colour diagram of NGC 6822 . It is thus possible that we see here interlopers which have magnitudes and colours similar to $\mathrm{C}$ stars.

Even though Galactic halo C stars can be well isolated with the SDSS colours (Margon et al. 2002; Downes et al. 2004), 


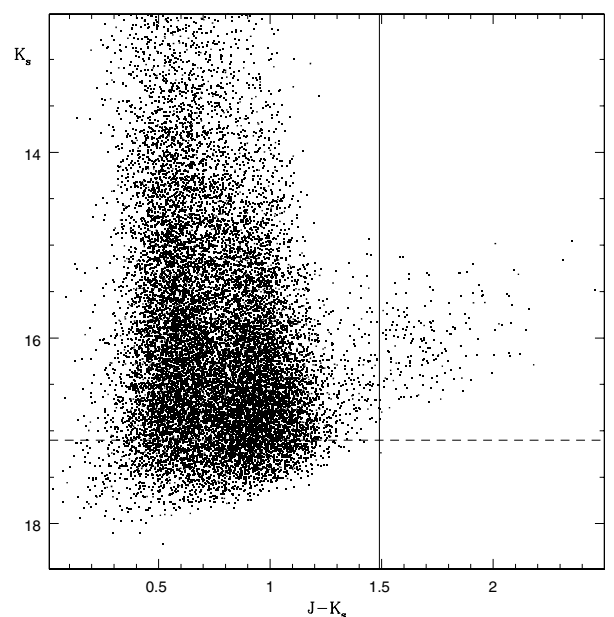

Fig. 2. Colour-magnitude diagram of the combined NE and SW fields. The vertical line marks the blue limit of the $\mathrm{C}$ stars region while the horizontal line indicates the magnitude of the TRGB.

C stars at $500 \mathrm{kpc}$ or more, with apparent magnitude $i^{\prime}=19$ or fainter can be confused with galaxies. Unfortunately, in the $K_{\mathrm{s}}$ vs. $\left(J-K_{\mathrm{s}}\right)$ colour-magnitude diagram, red galaxies can also pollute the $\mathrm{C}$ star region of the CMD when the magnitude limit is deep enough. The median $(J-K)$ colour of galaxies observed by Bershady et al. (1998), in the $17.5<K_{\mathrm{s}}<18.5$ range, is 1.73 . This colour is within our $\mathrm{C}$ star region but the magnitudes are, however, fainter than the NGC $6822 \mathrm{C}$ stars. Statistics from the first few fields of the MUNICS near infrared survey (Drory et al. 2001) indicate that there should be $\sim 400$ galaxies in our two CPAPIR fields within the magnitude interval: $15.25<K_{\mathrm{S}}<16.25$, bracketing well the $\mathrm{C}$ star region. Not all these galaxies are red enough, however, to be in the right colour interval. From the few colour data they published, we calculate that $11 / 56$, or $20 \%$ of those galaxies are redder than $(J-K)=1.49$, thus $80 \pm 35$ interlopers are expected among the candidate "C stars" of Fig. 2. These galaxies are bright enough to be in the not so distant Universe, most of them should therefore appear non stellar on our images.

DAOPHOT-II provides an image quality diagnostics SHARP. For isolated stars, SHARP should have a value close to zero, whereas for semi resolved galaxies and unrecognized blended doubles SHARP will be significantly greater than zero. On the other end, bad pixels and cosmic rays produce SHARP less than zero. SHARP must be interpreted as a function of the apparent magnitude of all objects because the SHARP parameter distribution flares up near the magnitude limit; see Stetson \& Harris (1998) for a discussion of this parameter. Since we want to investigate $\mathrm{C}$ star candidates, we select only stars within the magnitude interval of $\mathrm{C}$ stars: $14.5<K_{\mathrm{s}}<16.75$. The SHARP parameter distribution of some 10000 stars in this magnitude range is presented in the upper panel of Fig. 3. A Gaussian is fitted to the data. A slight surplus, above the Gaussian is seen for large positive SHARP values. Contrary to the bulk of stars, the 192 C star candidates have a SHARP distribution that confirms the presence of numerous non-stellar objects. We conclude from this figure that few genuine stars have SHARP larger than $\approx 0.30$.

For an alternative approach to the non-stellar pollution, we cross identify the CPAPIR database with the known $\mathrm{C}$ stars, identified from their $(R-I)$ and $(\mathrm{CN}-\mathrm{TiO})$ colour indices, by Letarte et al. (2002). Our two CPAPIR fields overlap a little with the CFH12k area. Sixty seven matches within $1.3^{\prime \prime}$ are found. The comparison of the SHARP parameter distributions for the

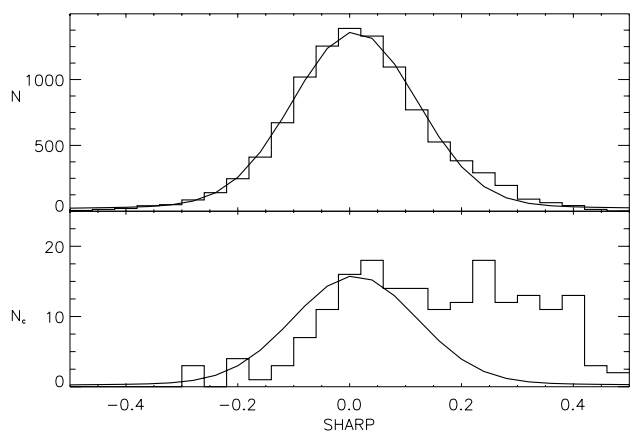

Fig. 3. A comparison of the SHARP distributions for stars in the AGB magnitude interval. All stars are shown in the top panel and the $\mathrm{C}$ star candidates in the lower panel. The Gaussian, from above, is scaled down and reproduced below.

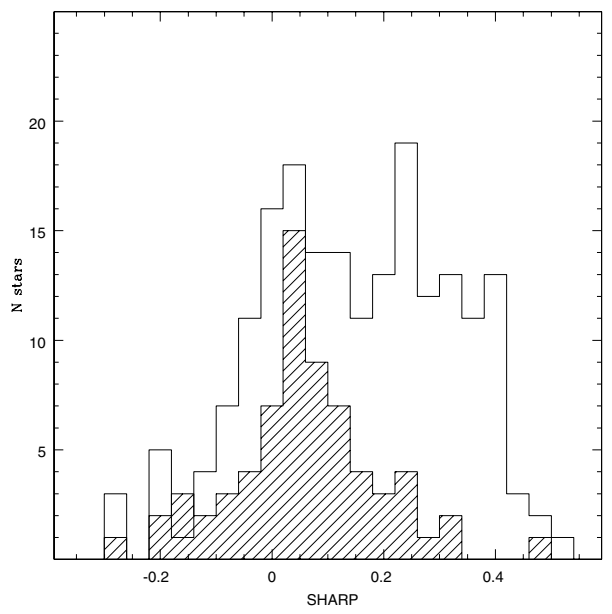

Fig. 4. SHARP distribution of the 192 candidate C stars compared to and the 67 matches to genuine $\mathrm{C}$ stars (shaded area). Barely $5 \%$ of the genuine $\mathrm{C}$ stars have a SHARP $>0.30$.

C star candidates, displayed in the lower panel of Fig. 3 is compared in Fig. 4 with the the SHARP distribution of genuine $\mathrm{C}$ stars. Figure 4 reveals that $95 \%$ of the $\mathrm{C}$ stars have a SHARP $<$ 0.30 , we adopt this upper limit for the definition of a stellar object. One must note that the pixel size of the CFH12k camera is $0.206^{\prime \prime}$, five times smaller than the CPAPIR pixel.

\subsection{The carbon stars}

There are then 142 stars with SHARP $<0.30$ and within the colour and magnitude range of $\mathrm{C}$ stars. If the rejected candidates are indeed galaxies, their number is within the uncertainty of the expected number of galaxies quoted in the previous section. The J2000.0 equatorial coordinates, magnitude and colour of the $\mathrm{C}$ stars are listed in Table 2. The numbering starts at 1001 in order not to confuse them with Letarte et al. (2002) list of some $900 \mathrm{C}$ stars previously discovered in NGC 6822. Their SHARP, as determined by DAOPHOT are also given in the last column. These stars are not all new discoveries because of the mentioned overlap between our fields and the CFH12k field. The 68 previously known C stars (Letarte et al. 2002) are marked with a $C$ while those found to be $\mathrm{M}$ stars, as explained in the next section, are marked with a $\mathrm{M}$. The Letarte et al. number is given in Col. 2, 000 is entered when no match is found. We calculate $\left\langle K_{\mathrm{s}}\right\rangle=16.02 \pm 0.41$, to be compared to $\left\langle K_{\mathrm{s}}\right\rangle=15.82 \pm 0.49$ for the sample of Kang et al. (2006) and $\left\langle K_{\mathrm{s}}\right\rangle=15.69 \pm$ 0.41 for the sample of Cioni \& Habing (2005). $\langle(J-K)\rangle$ are 
Table 2. C stars in the outer spheroid of NGC $6822^{a}$.

\begin{tabular}{ccccccccr}
\hline \hline$i d$ & $i d 2$ & RA & Dec & $K_{\mathrm{s}}$ & $\sigma_{K_{\mathrm{s}}}$ & $\left(J-K_{\mathrm{s}}\right)$ & $\sigma_{\left(J-K_{\mathrm{s}}\right)}$ & SHARP \\
\hline 1001 & 000 & $19: 42: 38.66$ & $-14: 50: 40.70$ & 16.379 & 0.054 & 1.782 & 0.089 & 0.03 \\
1002 & 000 & $19: 42: 43.66$ & $-14: 54: 35.00$ & 16.615 & 0.049 & 1.666 & 0.084 & -0.05 \\
1003 & 000 & $19: 42: 45.52$ & $-14: 53: 54.20$ & 16.540 & 0.059 & 1.720 & 0.093 & 0.17 \\
1004 & 000 & $19: 42: 48.95$ & $-15: 01: 15.40$ & 16.191 & 0.048 & 2.076 & 0.097 & -0.02 \\
1005 & 000 & $19: 42: 50.17$ & $-15: 03: 22.00$ & 16.427 & 0.068 & 1.636 & 0.087 & 0.22 \\
1006 & 000 & $19: 42: 52.92$ & $-15: 07: 32.00$ & 16.229 & 0.047 & 1.543 & 0.081 & -0.09 \\
1007 & 000 & $19: 43: 02.72$ & $-14: 45: 56.00$ & 16.377 & 0.060 & 1.994 & 0.106 & 0.14 \\
1008 & 000 & $19: 43: 04.89$ & $-14: 47: 35.70$ & 16.450 & 0.066 & 1.900 & 0.119 & 0.29 \\
1009 & 000 & $19: 43: 13.39$ & $-14: 42: 20.00$ & 16.541 & 0.069 & 1.614 & 0.101 & 0.29 \\
1010 & 000 & $19: 43: 17.65$ & $-14: 59: 26.10$ & 16.321 & 0.054 & 1.849 & 0.077 & -0.03 \\
1011 & 000 & $19: 43: 17.99$ & $-14: 58: 29.80$ & 16.290 & 0.057 & 2.182 & 0.098 & 0.20 \\
1012 & 000 & $19: 43: 18.60$ & $-14: 54: 02.10$ & 16.275 & 0.051 & 1.706 & 0.075 & 0.02 \\
\hline
\end{tabular}

a Table 2 is presented in its entirety in electronic form at the CDS. A portion is shown here for guidance regarding its form and content. Units of right ascensions are hours, minutes and seconds, and units of declination are degrees, arcminutes and arcseconds.

respectively, $1.74,1.90$ and 1.81 . We have $21 \mathrm{C}$ stars in common with the Cioni \& Habing (2005) list, the differences in mean magnitudes and mean colours are within the scatter. The comparison of their $K_{\mathrm{s}}$ magnitudes, taken at different epochs, fails to reveal a substantial population of variables among these $\mathrm{C}$ stars. For only two stars the $K_{\mathrm{s}}$ differ by more than $0.4 \mathrm{mag}$. One of them, with a $\Delta K_{\mathrm{s}}=0.47 \mathrm{mag}$, is a known $\mathrm{C}$ star for which we have its $I$ magnitude which can be compared with the measure of Cioni \& Habing (2005). Its $\Delta I=0.03 \mathrm{mag}$, certainly not suggestive of a variation. For the second star, the $\Delta K_{\mathrm{s}}$ amounts to $1.3 \mathrm{mag}$, a huge value for a Mira. However, its $\Delta I=0.06$ again ruling out substantial variation. We currently have an ongoing Mira survey in NGC 6822, within a year we should have sufficient observations to identify red variables.

\section{Discussion}

The identification of $\mathrm{C}$ stars from their NIR colours or from their $(\mathrm{CN}-\mathrm{TiO})$ index are not entirely equivalent, as Demers et al. (2002) have demonstrated. Our adopted $\left(J-K_{\mathrm{s}}\right)$ limit for C stars is not expected to yield a sample identical to the one obtained from other bands. In other words, some of our newly identified $\mathrm{C}$ stars might be outside the box of $\mathrm{C}$ stars in the $(R-I)$ vs. $(\mathrm{CN}-\mathrm{TiO})$ plane. To verify this possibility, we cross identify the new $\mathrm{C}$ stars with our full CFH12k database. Eighty five matches are found. These stars are plotted on the colour-colour diagram of Fig. 5. Sixteen stars must be classified M stars on the basis of their $(\mathrm{CN}-\mathrm{TiO})$ index. It turns out that the $\left(J-K_{\mathrm{s}}\right)$ colours of these stars are close to the blue limit with a $\left\langle\left(J-K_{\mathrm{s}}\right)\right\rangle=1.57$, while the $142 \mathrm{C}$ star candidates have $\left\langle J-K_{\mathrm{s}}\right\rangle=1.75$. A few more are marginal $\mathrm{C}$ stars very near the limit of acceptance.

Likewise, if we consider the M star region of the CMD we should expect to find some $\mathrm{C}$ stars among the $\mathrm{M}$ stars. Figure 6 presents the $(R-I)$ vs. $(\mathrm{CN}-\mathrm{TiO})$ of stars with observed $\left(J-K_{\mathrm{s}}\right)$ between 1.20 and 1.49 and matched to our CFH12k database. This is the region where $\mathrm{M}$ stars are found, see Cioni et al. (2004) for details. Of the 207 stars plotted less than $10 \%$ have $(\mathrm{CN}-\mathrm{TiO})$ corresponding to $\mathrm{C}$ stars. With a $\langle I\rangle=19.24$ these $19 \mathrm{C}$ stars have normal luminosity in $I$, their $\left\langle K_{\mathrm{s}}\right\rangle=16.21$ is, as expected, fainter than the $\left\langle K_{\mathrm{s}}\right\rangle$ of the redder $\mathrm{C}$ stars.

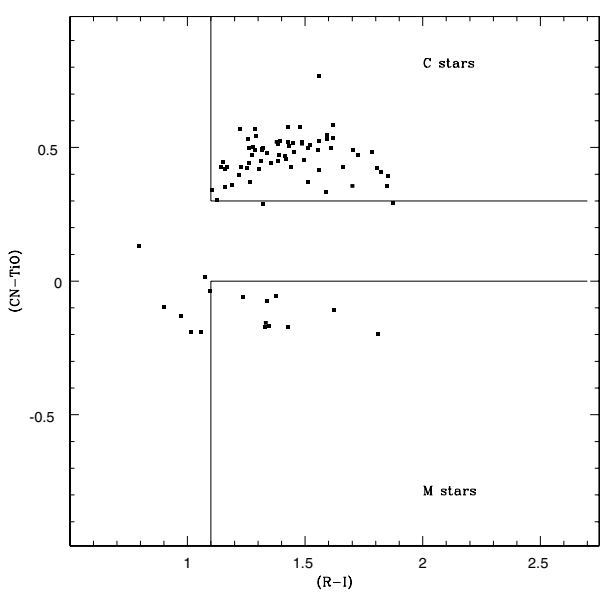

Fig. 5. Colour-colour diagram of $\mathrm{C}$ stars candidates matched to CFH12k database. Most stars with $\left(J-K_{\mathrm{s}}\right)$ colours close to the blue limit are actually M stars.

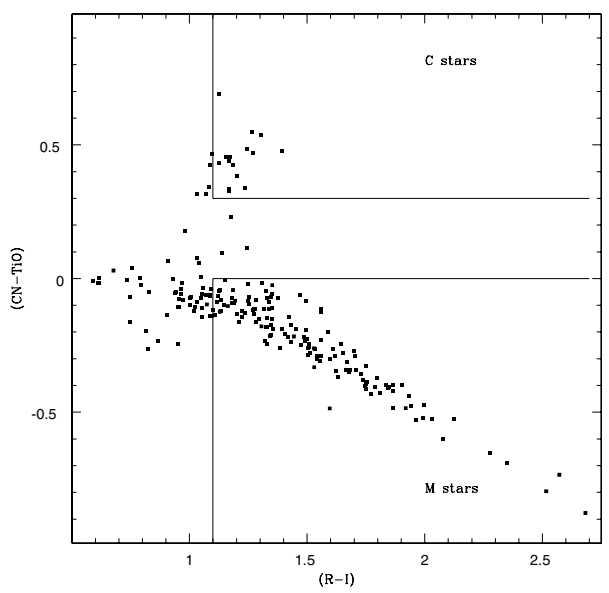

Fig. 6. Colour-colour diagram of $\mathrm{M}$ stars, selected from their $\left(J-K_{\mathrm{s}}\right)$ and matched to $\mathrm{CFH} 12 \mathrm{k}$ database. Less than $10 \%$ are actually $\mathrm{C}$ stars.

\subsection{SDSS colours of $C$ stars}

We can, using our MegaCam data (Battinelli et al. 2006) cross identify the $\mathrm{C}$ star candidates and obtain their $i^{\prime},\left(r^{\prime}-i^{\prime}\right)$ and $\left(g^{\prime}-r^{\prime}\right)$. We recover magnitude and colours for 136 of the 142 $\mathrm{C}$ stars, they are listed in Table 3 , where we give $i^{\prime},\left(r^{\prime}-i^{\prime}\right)$ 
Table 3. SDSS magnitude and colours of the $\mathrm{C} \operatorname{stars}^{a}$.

\begin{tabular}{lcccccc}
\hline \hline$i d$ & $i^{\prime}$ & $\sigma_{i}$ & $\left(r^{\prime}-i^{\prime}\right)$ & $\sigma_{r-i}$ & $\left(g^{\prime}-r^{\prime}\right)$ & $\sigma_{g-r}$ \\
\hline 1001 & 20.202 & 0.025 & 0.779 & 0.034 & 2.299 & 0.057 \\
1002 & 20.322 & 0.024 & 0.682 & 0.031 & 1.803 & 0.039 \\
1003 & 20.163 & 0.022 & 0.624 & 0.029 & 1.271 & 0.029 \\
1004 & 20.872 & 0.029 & 0.812 & 0.040 & 2.226 & 0.068 \\
1005 & 19.860 & 0.018 & 0.730 & 0.024 & 1.979 & 0.035 \\
1006 & 19.942 & 0.014 & 0.913 & 0.022 & 2.590 & 0.048 \\
1007 & 20.017 & 0.019 & 0.559 & 0.023 & 0.794 & 0.019 \\
1008 & 20.045 & 0.018 & 0.529 & 0.022 & 1.149 & 0.020 \\
1009 & 19.243 & 0.011 & 0.460 & 0.014 & 0.896 & 0.014 \\
1010 & 21.125 & 0.043 & 1.050 & 0.066 & 1.446 & 0.080 \\
1011 & 22.161 & 0.089 & 1.277 & 0.151 & 9.999 & 9.999 \\
1012 & 21.921 & 0.055 & 0.510 & 0.067 & 2.433 & 0.104
\end{tabular}

a Table 3 is presented in its entirety in electronic form at the CDS. A portion is shown here for guidance regarding its form and content.

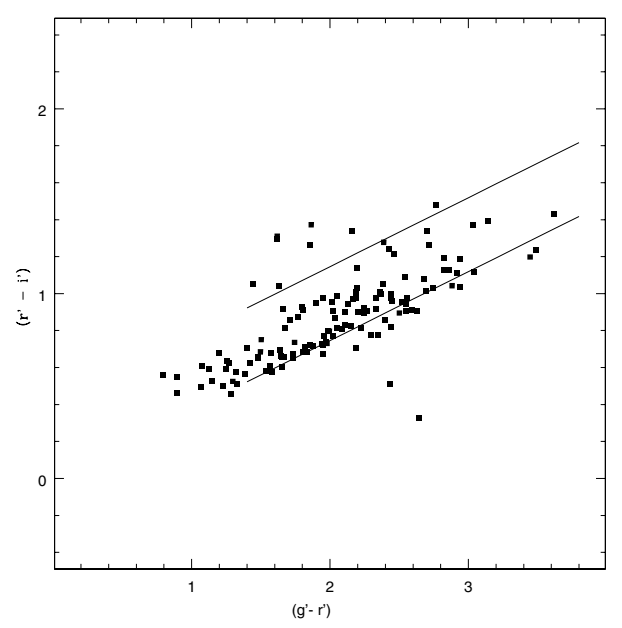

Fig. 7. Colour-colour diagram of $\mathrm{C}$ stars seen in the MegaCam database. The parallel lines limits the $\mathrm{C}$ star region, as defined from the unreddened colours, for NGC 6822 by Battinelli et al. (2006).

and $\left(g^{\prime}-r^{\prime}\right)$ along with their respective errors. Missing colours are entered as 9.999. We presume that the few missing stars fall in the gaps of the MegaCam mosaic. A few stars, not necessarily the faintest do not have $g^{\prime}$ magnitude. The stars of Table 3 are plotted on the colour-colour diagram presented in Fig. 7. The parallel lines define approximately the $\mathrm{C}$ star region of NGC 6822, as determined by Battinelli et al. (2006) from the dereddened colours of known $\mathrm{C}$ stars.

In Fig. 8 we compare the $i^{\prime}$ magnitude distribution of the known $\mathrm{C}$ stars with the newly discovered ones, shown by the shaded histogram. The $\left\langle i^{\prime}\right\rangle$ of the two sets are respectively 19.90 and 20.03. This essentially confirms that we are dealing with genuine $\mathrm{C}$ stars.

\subsection{Spatial distribution of $A G B$ stars}

Battinelli et al. (2006) have shown that C stars, as defined from their SDSS colours, can be traced in the spheroid of NGC 6822 to $\sim 40^{\prime}$. C stars, selected from their $\left(J-K_{\mathrm{s}}\right)$ colours, could be used to confirm this finding. Contrary to the MegaCam observations, the near infrared observations have not surveyed the whole spheroid. Cioni \& Habing (2005) observed the central $20^{\prime} \times 20^{\prime}$

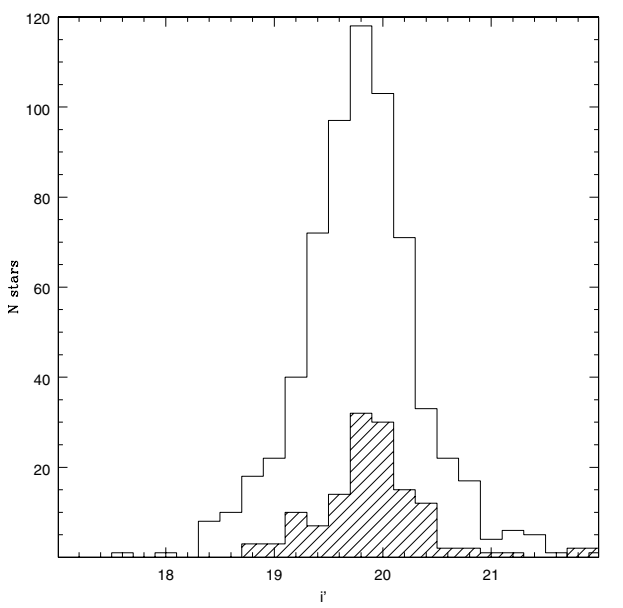

Fig. 8. Magnitude distributions of known $\mathrm{C}$ stars and of the newly discovered ones (shaded area). Both sets have essentially the same distribution.

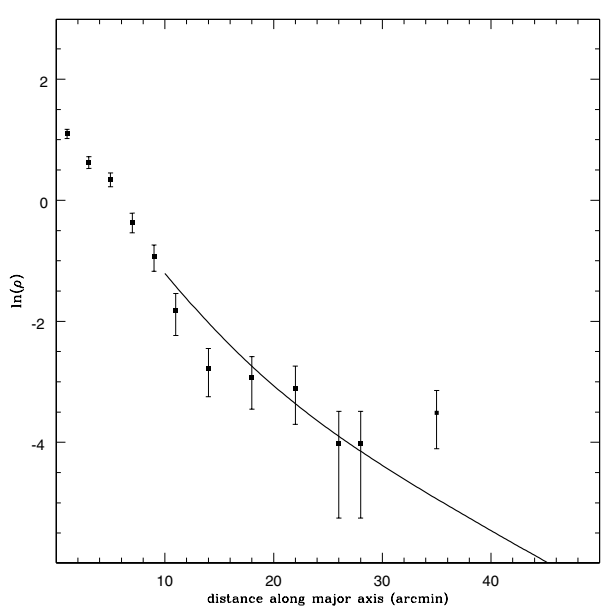

Fig. 9. Surface density profile of $\mathrm{C}$ stars along the major axis of the spheroid of NGC 6822. The solid line represents the double exponential profile established from RGB stars.

area of the galaxy while our two fields are aligned along the outer major axis. Nevertheless, a band of $\pm 7^{\prime}$ along the major axis of the spheroid, (at position angle of $65^{\circ}$ ) is fully sampled thus it could be used to determine the $\mathrm{C}$ star density profile along the major axis. We display, in Fig. 9, the surface density (stars per $\operatorname{arcmin}^{2}$ ) profile for $\mathrm{C}$ stars along the major axis. The solid curve, defined from $10^{\prime}$ to $50^{\prime}$, corresponds to the two-exponential profile determined from RGB star counts by Battinelli et al. (2006). It has been shifted down to match the points. Even if our figure is plagued by small number statistics, the outer bins contain just a few stars each, the $\mathrm{C}$ stars appear to follow the density of the bulk of the spheroid.

\section{Conclusion}

The main goal of the present paper was to find bright kinematic probes, namely $\mathrm{C}$ stars, to trace the rotation curve of the NGC 6822 spheroid up to its detected limit $\left(\approx 36^{\prime}\right)$. We have obtained accurate $J, K_{\mathrm{s}}$ photometry for stars in two $34^{\prime} \times 34^{\prime}$ fields located in the periphery of the spheroid along the major axis. On the basis of their $\left(J-K_{\mathrm{s}}\right)$ colours we find 142 bona fide $\mathrm{C}$ stars, half of them are newly discovered objects. Maps of these $C$ stars are shown in Fig. 10, for the NE field, and in Fig. 11 for the SW field. Solid dots represent known C stars while the new ones are 


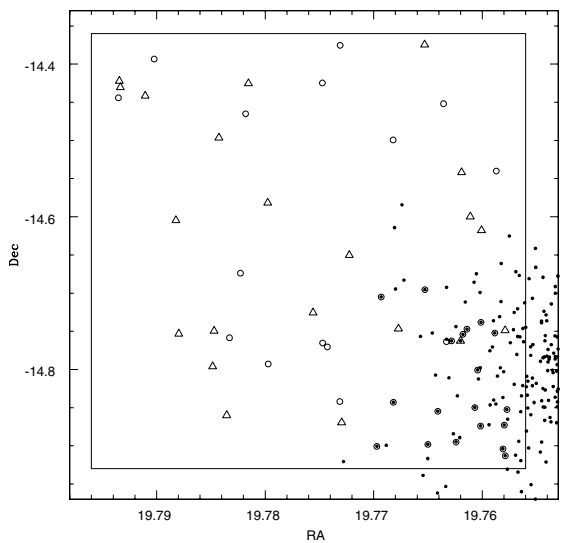

Fig. 10. Map of the NE CPAPIR field, $\mathrm{C}$ stars found in the CFH12k survey are shown by solid dots while the $\mathrm{C}$ stars identified here are represented by circles, for those with SHARP $<0.20$ and open triangles for stars with $0.20<$ SHARP $<0.30$.

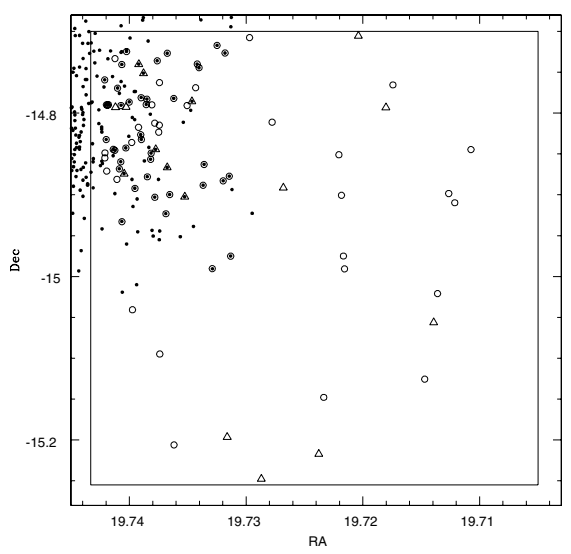

Fig. 11. Map of the SW CPAPIR field, C stars found in the CFH12k survey are shown by solid dots while the $\mathrm{C}$ stars identified here are represented by circles, for those with SHARP $<0.20$ and open triangles for stars with $0.20<$ SHARP $<0.30$.

indicated by open circles for those with SHARP $<0.20,(75 \%$ of them) and open triangles for those with $0.20<$ SHARP $<0.30$. As expected a few of the high SHARP objects match known C stars. However, one notes a unmistakable surplus of triangles in the areas corresponding to the outer spheroid. It is expected that a number of high SHARP candidates are non-stellar. Nevertheless, future observers can prioritize their candidates from the SHARP given. The inner square traces the border of the CPAPIR field. These new $\mathrm{C}$ stars will permit to more than double the length of the rotation curve of the spheroid and reach even further than the HI survey.

Acknowledgements. This research is funded in parts (S.D.) by the Natural Sciences and Engineering Research Council of Canada.

\section{References}

Artigau, E., Doyon, R, Vallée, P., Riopel, M., \& Nadeau, D. 2004, in Groundbased Instrumentation for Astronomy, ed. A. F. M. Moorwood, \& I. Masanori, Proc. SPIE, 5492, 1479

Barnard, E. E. 1885, A.N., 110, 125

Battinelli, P., Demers, S., \& Kunkel, W. E. 2006, A\&A, 451, 99

Bershady, M., Lowenthal, J. D., \& Koo, D. C. 1998, ApJ, 505, 50

Cioni, M.-R. L., \& Habing, H. J. 2005, A\&A, 429, 837

Cioni, M.-R. L., Habing, H. J., Loup. C., et al. 2004, The Messenger, 115, 22

Clementini, G., Held, E. V., Baldacci, L., \& Rizzi, L. 2003, ApJ, 588, L85

Davidge, T. J. 2003, PASP, 115, 635

Demers, S., Dallaire, M., \& Battinelli, P. 2002, AJ, 123, 3428

Demers, S., Battinelli, P., \& Kunkel, W. E. 2006, ApJ, 636, L85

Downes, R. A., Margon, B., Anderson, S. F., et al. 2004, AJ, 127, 2838

Drory, N., Feulner, G., Bender, R., et al. 2001, MNRAS, 325, 550

Elias, J. H., \& Frogel, J. A. 1985, ApJ, 289, 141

Hodge, P. W. 1977, ApJS, 33, 69

Hodge, P., Smith, T., Eskridge, P., MacGillivray, H., \& Beard, S. 1991, ApJ, 379, 621

Hubble, E. P. 1925, ApJ, 62, 409

Kang, A., Sohn, Y.-J., Kim, H.-I., et al. 2006, A\&A, in press

Komiyama, Y., Okamura, S., Yagi, M., et al. 2003, ApJ, 590, L17

Lee, M. G., \& Hwang, N. 2005, in Near-fields cosmology with dwarf elliptical galaxies, ed. H. Jerjen, \& B. Binggeli (Cambridge: CUP), IAU Symp., 198, 181

Letarte, B., Demers, S., Battinelli, P., \& Kunkel, W. E. 2002, AJ, 123, 832

Margon, B., Anderson, S. F., Harris, H. C., et al. 2002, AJ, 124, 1651

Pietrzyński, G., Gieren, W., Udalski, A., et al. 2004, AJ, 128, 2815

Schlegel, D., Finkbeiner, D., \& Davis, M. 1998, ApJ, 500, 525

Stetson, P. B. 1994, PASP, 106, 250

Stetson, P. B., \& Harris, W. E. 1988, AJ, 96, 909

van den Bergh, S. 2000, The Galaxies of the Local Group, Cambridge Astrophysics Series, 35

Weldrake, D. T. F., de Blok, W. J. G., \& Walter, F. 2003, MNRAS, 340, 12 